\title{
Hochkonzentriertes Phytotherapeutikum zugelassen
}

- Sinupret $^{\circledR}$ extract ist ein Meilenstein in der Weiterentwicklung des rezeptfreiem Erkältungsmittels Sinupret ${ }^{\oplus}$. Durch ein patentiertes Trockenextraktverfahren gelingt es, aus der im Vergleich zu Sinupret ${ }^{\oplus}$ forte vierfachen Drogenmenge die wirksamkeitsbestimmenden Inhaltsstoffe der 5-PflanzenKombination, bestehend aus Schlüsselblumenblüten, Ampferkraut, Holunderblüten, Enzianwurzel und Eisenkraut, stark aufzukonzentrieren: Sinupret ${ }^{\circledR}$ extract weist im Vergleich zu Sinupret ${ }^{\oplus}$ forte $z$. B. eine dreibis vierfach höhere Konzentration an Bioflavonoiden auf. Für diese Wirkstoffgruppe konnte bereits mit Sinupret ${ }^{\oplus}$ eine dosisabhängige Steigerung der Chloridionen-Sekretion am respiratorischen Epithel, und somit eine dosisabhängige Steigerung von Sekretolyse bzw. mukoziliärer Clearance, nachgewiesen werden [Virgin F et al. The Larynoscope 2010;120(5):1051-1056].

\section{Dosisabhängige Wirkung bestätigt}

Aktuelle pharmakologische Daten bestätigen eine dosisabhängige Wirkung und die sekretolytische, antiinflammatorische und antivirale Wirksamkeit des Trockenextrakts im Versuchsansatz [Kreindler J et al. The American Journal of Rhinology and Allergy 2012;26(6):439-443; Rossi A et al. Fitoterapia 2012;83(4):715-720; Glatthaar-Saalmüller B et al. Phytomedicine 2011;19:1-7]. Sinupret ${ }^{\oplus}$ extract löst besonders effektiv das zähe Sekret, öffnet die Nase und befreit vom Druckkopfschmerz.

Die klinische Wirksamkeit und die hohe Verträglichkeit des neuen Phytopharmakons konnten auch am Patienten in Zulassungsstudien belegt werden. Die Studienergebnisse der multizentrischen, doppelblinden, randomisierten, placebokontrollierten klinischen Parallelgruppenstudien zur Untersuchung von Wirksamkeit und Sicherheit des neuen Sinupret ${ }^{\circledR}$ extract bei akuter Rhinosinusitis wurden bereits im letzten Jahr veröffentlicht [Jund $\mathrm{R}$ et al. Rhinology 2012;50(4):417-426].

Die neue 5-Pflanzen-Kombination steht in bundesdeutschen Apotheken seit Okto- ber 2012 zur Therapie akuter, unkomplizierter Entzündungen der Nasennebenhöhlen für Patienten ab 18 Jahren zur Verfügung. Bionorica SE hat bereits im Jahr 2012 den Antrag gestellt, Sinupret ${ }^{\circledR}$ extract auch in ihren internationalen Kernmärkten zuzulassen.

Die Relevanz des neuen Phytopharmakons hat die Bionorica SE im Vorfeld der Zulassung in einer bundesweit angelegten Marktforschung getestet. Befragt wurden Allgemeinmediziner, HNO-Ärzte, Apotheker und pharmazeutisch-technische Assistenten (PTA). Nahezu alle Teilnehmer bestätigten, dass das Phytopharmakon neue Maßstäbe setzt und eine Therapielücke in der Indikation Rhinosinusitis geschlossen wird. Die Einführung des Präparates wurde mit dem erweiterten Angebot einer interaktiven und weborientierten Fortbildung für Ärzte, Apotheker und PTAs verbunden, die seit dem 1. Oktober online ist.

Die Forschungs- und Entwicklungsphilosophie des Unternehmens hat bislang zu 16 Phytopharmaka in den Bereichen Atemwege, Urologie, Gynäkologie und Schmerz geführt. Die mit weltweit fast 500 führenden Universitäten und Kliniken kooperierende und mit einem hohen Aufwand forschende Bionorica SE fokussiert sich da-

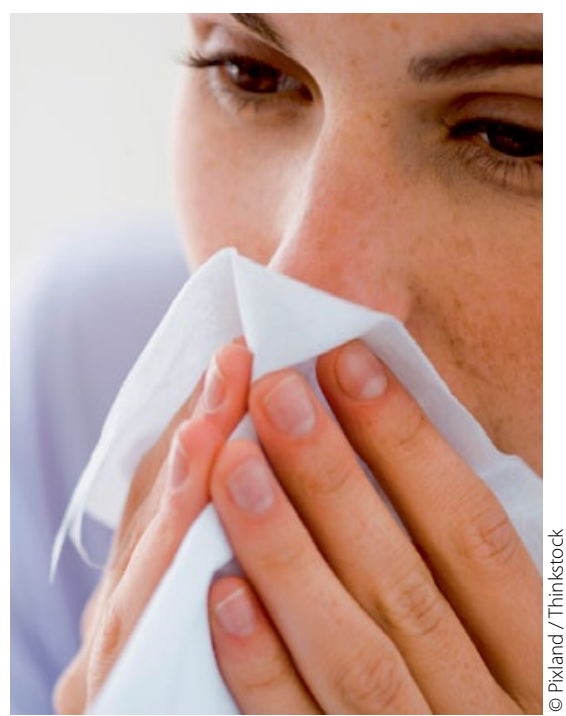

Ihr kann jetzt schneller und besser geholfen werden.

rauf, die Wirkung und Nebenwirkungsarmut ihrer pflanzlichen Arzneimittel mit international anerkannten pharmakologischen und klinischen Studien zu belegen. Schon Ende des Jahres wird die Bionorica SE zusammen mit der Bundesrepublik Österreich und dem Land Tirol ein weiteres Forschungsinstitut am Universitätsstandort Innsbruck eröffnen, um in relativ kurzer Zeit neue pflanzliche Wirkstoffe zu identifizieren und daraus Phytopharmaka für bestimmte Indikationen entwickeln zu können.

- Red.

Quelle: nach Informationen von Bionorica SE

\section{Kurz notiert}

Rachenbefeuchtung lindert Reizhusten

- Bei hartnäckigem Reizhusten kann ein Therapieversuch mit Halstabletten lohnen, die die Mund- und Rachenschleimhaut mit einem Feuchtigkeitsfilm überziehen, erklärte der HNO-Facharzt Dr. Thorsten Zehlicke vom Bundeswehrkrankenhaus Hamburg. „Trockene Schleimhäute sind bei vielen Patienten die Ursache eines Reizhustens", erläuterte Zehlicke.

Mit den hyaluronhaltigen GeloRevoice ${ }^{\circledast}$ Halslutschtabletten gelingt es, die Schleimhaut jeweils eine Stunde lang effektiv zu befeuchten. Über die optische
Kohärenztomografie konnte in seiner Klinik nachgewiesen werden, dass sich nach Lutschen der Halstabletten tatsächlich ein Feuchtigkeitsfilm auf den Schleimhaut bildet.

In einer offenen Anwendungsbeobachtung wurden 39 Patienten mit Reizhusten sieben bis zehn Tage lang mit GeloRevoice ${ }^{\circledR}$ behandelt. 26 Patienten waren nach zwei Wochen vollkommen beschwerdefrei.

- Dirk Einecke

Quelle: Pressekonferenz „Neue klinische Untersuchungen zu GeloMyrtol ${ }^{\circledR}$ forte und GeloRevoice ${ }^{\oplus}$, Hamburg, Januar 2013 (Veranstalter: Pohl Boskamp) 